\title{
UACH, UADY y LA SALLE Chihuahua: un análisis de los ámbitos de dominio de la competencia comunicativa para la generación de productos académicos
}

\author{
Lourdes Elizabeth Carrillo Vargas \\ Servicios Educativos de Gobierno del Estado, \\ Departamento de Investigación Educativa, \\ Universidad Autónoma \\ ecarrillo@uach.mx \\ elizabethbnt@hotmail.com
}

\begin{abstract}
Resumen
La Universidad Autónoma de Chihuahua, la Universidad Autónoma de Yucatán y la Universidad de Lasalle de Chihuahua fueron el contexto elegido para realizar la investigación de los ámbitos de la competencia comunicativa y sus ámbitos de dominio en la generación de productos académicos; el tema de las competencias en las instituciones de Educación son permeables en los procesos de transformación, motivo por el cual el académico adquiere el compromiso de convertirse en agente de cambio, en migrante a las nuevas tecnologías, a los diversos medios de difusión que rebasan el formato impreso y sobre todo el obtener la habilidad de discernir para elegir información, analizarla y reestructurarla para posteriormente construir o generar nuevo conocimiento. La competencia comunicativa se encuentra inmersa en las diversas categorizaciones sobre las competencias, los organismos internacionales como la UNESCO, OCDE, BID, entre otros coinciden que esta competencia es clave en este mundo globalizado. Los académicos de la Universidad Autónoma de Chihuahua así lo refieren y se logra establecer una categorización sobre los productos existentes, los criterios de calidad que se utilizan, los diversos circuitos para su difusión y los ámbitos de dominio de la competencia comunicativa que se ponen en juego para su elaboración.
\end{abstract}

\section{Palabras clave}

Info competencia, tecno competencia, media competencia, adquisición de lenguajes, ámbitos, dominios.

\section{Introducción}

Esta investigación nace de un proceso de análisis descriptivo, a través del devenir histórico educativo, su conceptualización ha cambiado, actualmente, la formación y preparación refleja una prioridad para el ser humano, cualquier actividad debe realizarse de la mejor manera, mantener un alto nivel de eficacia y eficiencia; en ámbito educativo, la responsabilidad es mayor, somos la base formativa de la sociedad, en nuestras manos tenemos la responsabilidad de la formación de nuestros niños, jóvenes que en un futuro serán la gente productiva que propiciara el cambio de nuestro país.

La política educativa nos advierte sobre las deficiencias encontradas y nos marca las pautas emergentes a seguir con la finalidad de lograr un cambio, eficientar 
y reformar los diversos ámbitos en educación, nos habla sobre los cambios curriculares, la profesionalización de los docentes y la ruptura de paradigmas establecidos. El termino competencia se convierte en agente de cambio en las universidades; la competencia comunicativa como piedra angular en todas las clasificaciones y sus ámbitos de dominio considerados necesarios para insertase en este mundo globalizado y tecnológico. Esta investigación toma en consideración a tres universidades en donde se determinan los ámbitos de la competencia comunicativa para la generación de nuevo conocimiento y su difusión en diversos circuitos.

\section{Universidades en donde se realiza la investigación}

Universidad Autónoma de Chihuahua

La Universidad Autónoma de Chihuahua (UACH), es una institución de educación superior, pública, autónoma, dedicada a formar individuos con valores universales, capaces de dar respuestas pertinentes y creativas ante un mundo en constante cambio. En este sentido, el universo de este estudio contempló a los profesores de tiempo completo (648), considerando que a ellos pertenecen las obligaciones académicas de la Universidad: docencia, tutoría, gestión e investigación, sin embargo, mediante un análisis reflexivo y considerando que la finalidad de la investigación está vinculada con la generación de conocimiento se reconsidero la muestra a los académicos de la Universidad que pertenecen al perfil PROMEP (295) y/o SNI (67). Según datos estadísticos del 2009 de la UACH.

\section{Universidad Autónoma de Yucatán: Facultad de Educación}

La Facultad de educación de la
Universidad Autónoma de Yucatán promueve el desarrollo del conocimiento y el mejoramiento de la educación en México a través de: La docencia, extensión de la cultura y la divulgación del conocimiento en beneficio de la sociedad, desarrollo de la tecnología educativa, presentación de servicios asociados a la educación, desarrollo de sistemas para la planeación y administración de la educación y el diseño desarrollo y evaluación curricular. La Facultad de Educación campus Mérida y Tzimin; ambos con académicos profesionales y comprometidos con su desempeño dentro de la Universidad; fueron 25 los académicos que colaboraron para esta investigación.

\section{Universidad La Salle de Chihuahua}

La Universidad de la Salle en su constante transformación, instaura un proceso de capacitación continua para fortalecer la actualización de los docentes, es una universidad que se declara como de inspiración cristiana con carisma lasallista y postula como características distintivas: busca la verdad y proporciona los conocimientos necesarios para lograr que sus estudiantes sean profesionistas de la más alta calidad; de inspiración cristiana, propone valores no en abstracto, sino desde la práctica de los mismo con carisma lasallista es heredera y fiel continuadora de una práctica sin interrumpir de excelencia educativa durante 331 años y en más de 85 países. En el ciclo escolar enero- junio del 2012 la universidad contaba con 224 docentes que con su testimonio sobre las competencias académicas fortalece la recolección de datos para esta investigación.

\section{Las competencias en el sistema educativo}

La nueva Universidad inicia con un 
proceso de transformación e innovación a principios de este siglo, el modelo pedagógico tradicionalista es modificado a un modelo centrado en el aprendizaje basado en competencias. Menciona Marín (2003) que: "el modelo educativo por competencias se sustenta en el enfoque holístico, enfatizado en el desarrollo constructivista de conocimientos, habilidades, actitudes $y$ valores que permita a los estudiantes insertarse adecuadamente en la estructura laboral y adaptarse a los cambios y reclamos sociales". Esta metamorfosis del sistema educativo tiene como sistema medular el desarrollo de competencias" ¿Pero que es una competencia?; el concepto de competencia es considerado desde una perspectiva holística y no como el resultado de la simple aritmética de conocimientos, actitudes y habilidades. Se trata de un concepto integrador, en donde no basta con considerar uno o más de los elementos por separado, sino la articulación de ellos. De igual manera se puede considerar un conjunto de conocimientos, destrezas, habilidades $\mathrm{y}$ actitudes que posibilitan al individuo su utilización en situaciones diversas, que poseen cualidades para múltiples aplicaciones, que tienen diferentes usos, es decir, más amplia posibilidad de transferencia. Con todo, aunque no exento de polémica, el modelo de competencias ha logrado imponerse como paradigma en la redefinición de los objetivos de la educación superior. La perspectiva de la educación universitaria según el modelo por competencias, empieza por los profesores, en la prevención de que eventualmente permee hacia los estudiantes.

Entre las innumerables propuestas de clasificación de competencias, sobresalen los dominios referidos a la comunicación. Esta actividad suele estar presente en todos los contextos y circunstancias humanas y es base de cualquier proceso de conocimiento e interacción de los individuos, se constituye en el ambiente natural de las personas que, consciente $\quad 0 \quad$ inconscientemente, voluntaria e involuntariamente, intercambian constante y permanentemente mensajes, tanto de manera directa como de forma indirecta a través de infinidad de mecanismos y medios. Por consiguiente, el Docente Universitario es un vínculo importante en el proceso educativo y la Educomunicación.

Por Edu-comunicación se entiende "el proceso que busca informar y formar en el sujeto un conjunto de dimensiones educativas que empiezan por la alfabetización tecnológica, informativa y mediática" (Martínez De Toda y Terrero, 1999).

Blazquez Entonado (2001) menciona: "El papel de los docentes es más importante cada día en los nuevos entornos culturales y educativos que se están creando y que se deben crear con la ayuda de las Tecnologías de la Información y la Comunicación. La innovación metodológica y de profusión y enriquecimiento de las actividades docentes son ejes de orientación clara hacia un mundo en construcción". El docente se ve sometido a un proceso de des aprendizaje y de reaprendizaje; a la ruptura de paradigmas establecidos para la formación de un nuevo andamiaje educativo que actualice y fortalezca su quehacer pedagógico.

Al integrar el modelo de competencias el docente adquiere un rol propositivo, facilitador e integrador de los procesos de enseñanza, al mismo tiempo se convierte en generador activo de nuevos conocimientos para los cuales es necesario posea la competencia de 
comunicación.

\section{La competencia Comunicativa}

Elena Cano (2007) califica de instrumental y transversal a la competencia comunicativa y la identifica más bien en términos de 'capacidad', lo mismo hace con la utilización de TIC, la cual sitúa como una competencia por separado, es decir, como dos competencias distintas y complementarias, nosotros creemos que la competencia en TIC se subordina como domino a la competencia comunicativa, pero igualmente es, para ella, una capacidad instrumental. Vincula, así mismo, la competencia en el manejo de información o 'Info-competencia' subsumiéndola en la competencia en el uso de tecnologías, o 'tecno-competencia'. No obstante, tiene razón cuando afirma que: "Al incorporar nuevas tecnologías a nuestra vida, se producen cambios técnicos. Aquellos que nos resultan realmente sustantivos nos llevan a cambios pedagógicos y a cambios en nuestro quehacer profesional" (Cano, 2007, pág. 144).

Numerosos estudios relacionados con el Proyecto Tuning (2007), versan sobre el estudio de las competencias genéricas y las competencias específicas, las genéricas han sido también calificadas de transversales; transferibles a multitud de funciones y tareas, y las Competencias Específicas se relacionan directamente con la ocupación; en donde la competencia comunicativa es analiza y descrita específicamente.

La competencia comunicativa ostenta diversos dominios, que varían en cuanto a exigencia y complejidad en el establecimiento y desarrollo de competencias. Para un académico universitario $\mathrm{y}$, específicamente en la identificación y valoración de sus habilidades y capacidades para la generación de productos de calidad, que bien pueden caber en un amplio catálogo e incidir en diferentes ámbitos, desde su grupo de alumnos, en primera instancia hasta el sistema económico productivo y sociocultural como instancias de mayor alcance y acotado por el marco de la competencia comunicativa, al menos cuatro elementos quedan englobados en este término establecida por Cortés (2008) :

a) Competencia adquisición de lenguajes

b) Tecno-competencia

c) Info-competencia

d) Media-competencia

Cada uno de estos ámbitos de la competencia comunicativa es analizado en esta investigación en relación a la producción académica de los docentes universitarios y los diversos dominios existentes entre ellos.

En las competencias académicas se conforman nuevos roles del Docente universitario, involucran una transformación en educación, el modelo por competencias instaura está necesidad $\mathrm{y}$ es concerniente a la competencia de comunicación la vinculación del proceso de la generación de productos académicos. Se requiere el dominio de la Info competencia, tecno competencia, adquisición de nuevos lenguajes y media competencia como cimiento de inserción a la innovación educativa.

\section{Metodología}

La investigación realizada en etapas: primeramente, una búsqueda de información documental para fortalecer y fundamentar la investigación, realización de grupos focales, entrevistas a Maestros, directores y secretarios académicos de diversas Facultades, aplicación de una 
encuesta en formato digital e impreso fueron los instrumentos utilizados para la recolección de la información.

En una segunda etapa por medio de análisis y categorización de información se estructuran los resultados obtenidos Esta investigación culmina con una serie de propuestas de mejora para establecer y dar a conocer los ámbitos de la competencia comunicativa y su dominio en la generación de conocimiento.

\section{Resultados}

De inicio coinciden en una clasificación de la producción académica la cual clasifican en: dentro del aula, como gestor y como investigador

\begin{tabular}{|c|c|}
\hline \multicolumn{2}{|c|}{ Dentro del Aula } \\
\hline $\begin{array}{l}\text { Los académicos universitarios realizan } \\
\text { diversos materiales que utilizan en su práctica } \\
\text { docente, la comparten con sus alumnos y } \\
\text { colegas pero solo transitan dentro del aula }\end{array}$ & $\begin{array}{l}\text { Presentaciones power point } \\
\text { Antologías } \\
\text { Monografias } \\
\text { Cuadros de doble entrada } \\
\text { Material didáctico } \\
\text { Videograbaciones, } \\
\text { Objetos de aprendizaje } \\
\text { Programas de asignaturas }\end{array}$ \\
\hline \multicolumn{2}{|l|}{ Tabla 1Producción académica dentro del aula } \\
\hline \multicolumn{2}{|c|}{ Como Gestor } \\
\hline El académico dentro de sus obligaciones como & Reportes de estudiantes \\
\hline $\begin{array}{l}\text { gestor y tutor, genera diversos productos en } \\
\text { apoyo a sus estudiantes y a la administración } \\
\text { de la Universidad. Su forma de circulación es } \\
\text { solo en la Universidad e instituciones } \\
\text { vinculadas a algún proceso educativo }\end{array}$ & $\begin{array}{l}\text { Supervisados en servicio social y Prácticas } \\
\text { profesionales } \\
\text { Diseño curricular } \\
\text { Planes institucionales }\end{array}$ \\
\hline
\end{tabular}

Tabla 2 Producción académica como Gestor

\section{Como Investigador}

El académico dedicado a la investigación culmina en diversos productos, a través de ellos fortalece el ámbito disciplinario al que se dedica y con ello a la Educación

Tabla 3 Producción Académica como investigador
Monografías, Proyectos de mejora profesional, Catalogo e índices, Textos divulgación Artículos científicos, Libros, Capítulos de libros, Obra artística: plástica, musical, danza, teatro, Obra de ingeniería, Patentes,

Traducciones técnicas, Desarrollo de software Manuales, Banco y bases de datos, Memorias de congreso, Ponencias en congreso, Conferencia magistral, Ensayos, Programas tv y radio, Guiones técnicos, Compilación de libros, Reseñas, Dictamen de productos científicos, Dictamen de proyectos científicos 
A partir de esta clasificación y analizada de forma continua dentro los diferentes grupos de expertos establecieron diversos criterios de calidad para los productos académicos y su clasificación:

a) Ser claro, entendible, objetivo, concreto y especifico y pasar por un proceso de validación.

b) Estar avalados por la academia del área y/o academia institucional

c) Material específico entendible para cualquier lector

d) El impacto del programa sobre la sociedad, sustentado en la identificación de una oportunidad de mejora en el campo profesional. Factible de aplicación con un funcionamiento efectivo, eficaz y con facilidad de utilizarse.

e) De acuerdo a criterios internacionales

f) Sometidos a arbitraje con resultados positivos

g) Registrado con un ISBN, registrado en INDEAUTOR

h) Se consideran modelos de utilidad los objetos, utensilios, aparatos o herramientas que, como resultado de una modificación en su disposición, configuración, estructura o forma, presenten una función diferente respecto de las partes que lo integran o ventajas en cuanto a su utilidad.

i) Requieren un alto nivel de destreza y conocimiento por parte de la persona de la terminología tratada. Los criterios de calidad son determinados dependiendo del área disciplinar a la que correspondan y al órgano evaluador al que son sometidos.

\section{Ámbitos de dominio de la competencia comunicativa}

Coincidentemente los académicos

universidades analizadas especifican como necesarios diversos dominios en los ámbitos de la competencia comunicativa definidos de la siguiente manera:

\section{Adquisición de lenguajes}

a) Capacidad de comunicación verbal en forma efectiva

b) Capacidad de comunicación escrita en forma efectiva

c) Habilidades de lectura e interpretación de textos científicos

d) Habilidad a un segundo idioma; leer, escribir, escuchar y hablar

e) Habilidad en redacción científica buen uso y manejo de la información

f) Comunicación efectiva oral y escrita en función del contexto

g) Utiliza el diseño experimental en el planteamiento de proyectos de investigación

\section{Info competencia}

a) Elaboración de propuestas de investigación, a partir de la identificación y análisis de problemas científicos, apoyado en el uso eficiente de las fuentes documentales

b) Análisis y reflexión los diferentes componentes de un problema y sus interrelaciones

c) Elaboración, interpretación, adecuación y aplicación de reportes de investigación con base a resultados obtenidos

d) Identificación de necesidades problemáticas en el estado de 
conocimiento

e) Localizar, analizar y calificar información científica aplicando los principios para su organización

f) Contextualización de los productos a la cultura

g) Planear, analizar y abstraer información en diversos escenarios a la luz del conocimiento

h) Habilidad en manejo de información para determinar las ideas

i) Análisis para identificar el origen de situaciones problemáticas de fenómenos potencialmente estudiables

j) Adapta críticamente sus propios conceptos y comportamientos a normas, ambientes y situaciones cambiantes.

\section{Media Competencia}

a) Optimiza y enlaza recursos disponibles

b) Propicia la gestión de recursos

c) Conoce, adapta y manipula correctamente las tecnologías

d) Demuestra un buen dominio en el manejo de recursos documentales y electrónicos que apoyan la búsqueda de información científica.

e) Uso y manejo de programas computacionales para la presentación de sus trabajos escritos, así como de apoyo audiovisual en la exposición de temas

f) Preparación y presentación oral de sus trabajos apoyándose en materiales audiovisuales incluyendo conclusiones estadísticas cuando así lo requiera

g) Maneja herramientas tecnológicas metodológicas de investigación

h) Aplica la tecnología disponible a la solución de problemáticas

\section{Tecno Competencia}

a) Determina los requerimientos de los diversos circuitos mediáticos para su utilización

b) Localiza fuentes de información de calidad en su área de conocimiento.

c) Realiza análisis de impacto de las aportaciones al estado del conocimiento

d) Conocimiento de circuitos mediáticos

e) Optimiza y enlaza recursos disponibles

f) Identificación de medios de publicación

g) Comunica efectivamente los resultados de sus investigaciones, con participación en eventos académicos y científicos

\section{Conclusiones}

Los académicos universitarios inmersos en un mundo globalizado se convierten en migrantes hacia la era mediática, la universidad se encuentra en constante transformación y el académico como un precursor de este proceso; las nuevas tecnologías, los circuitos mediáticos, los nuevos lenguajes técnicos y científicos y el portal infinito de información existente, reclaman que la competencia comunicativa y sus ámbitos de dominio como son: Info competencia, tecno competencia, media competencia y la adquisición de lenguajes estén presentes en el académico universitario a través de un constante dominio que permita insertarse al siglo XXI $y$ sus requerimientos. Las universidades 
analizadas con sus características particulares refieren una misma preocupación: La transformación docente continua que coadyuve al desarrollo de su universidad para ofertar una educación de calidad.

\section{Referencias}

Blazquez, E. F. (2001). Las organizaciones que enseñan las organizaciones que aprenden. Revista de Educación ISSN0213 No 20 , 47-64.

Blazquez, E. F. (2001). Sociedad de la Información y Educación. Junta de Extremadura: Mérida: Consejería de Educación, Ciencia y Tecnología.

Cano, E. (2007). Como mejorar las competencias de los docentes: guía para la autoevaluación y el desarrollo de las competencias del profesorado. Barcelona: GRAO.

Cortés, J., \& Carrillo, L. (2012). La Competencia comunicativa en la producción académica. En E. Cisneros, B. García, E. Luna, \& R. Marín, Evaluación de competencias
Docentes en Educación Superior (págs. 249-282). México: JP.

Marín Uribe, R. (2006). El modelo Educativo de la UACH: elementos para su construcción. Chihuahua: Dirección de extensión y Difusión Cultural.

Martínez de Toda y Terrero, J. (1999). Las seis dimensiones de la educación para los medios (Metodología de evaluación). Roma: Universidad Gregoriana, Italia.

Tuning de América Latina. (2007). Reflexiones y Perspectivas de la Educación Superior en América Latina. España: Universidad Deusto.

UACH. (2008). Organización de la UACH. Recuperado el 7 de noviembre de 2010, de http://www.uach.mx/institucional _y_juridica/2008/03/07/organizac ion/

UACH, D. A. (2000). Reforma y Flexibilidad. Chihuahua, Chih: UACH. 